
(مطالعه موردى: فولاد محلهِ سمنان)

\author{
هانيه ببرنزاد زيارت '، على اصغر ذوالفقارى '، محمدرضا يزدانى '، على اصغر هاشمى و و محمدكيا كيانيان

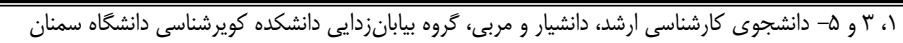

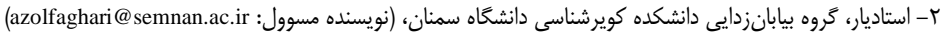

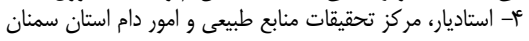

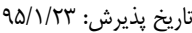

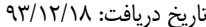

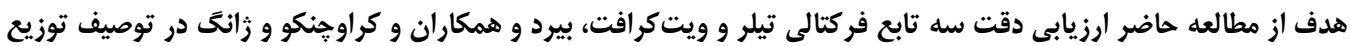

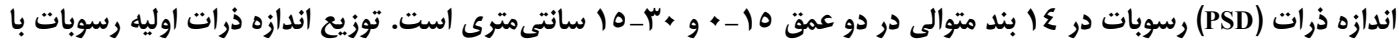

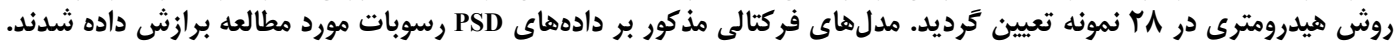

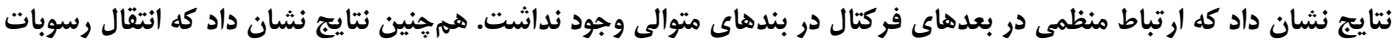

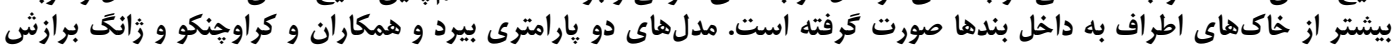

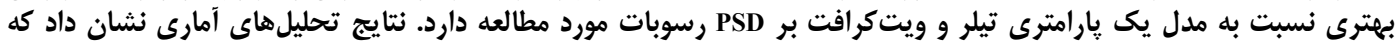

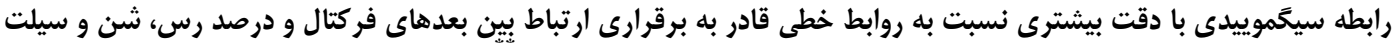

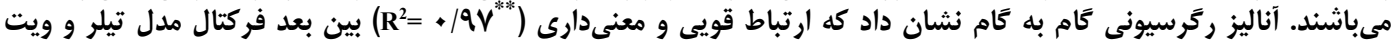

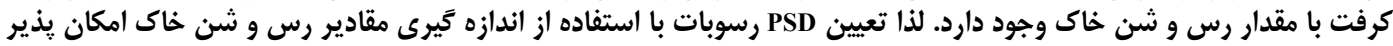

مىباشد.

وازههاى كليدى: ابعاد فر كتال، بندهاى رسوبَّير، رابطه سيگموييدى

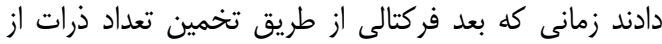

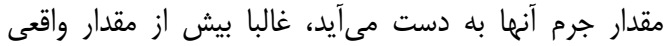

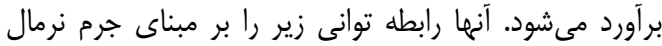

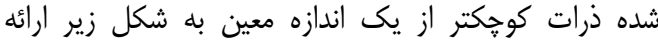

$\frac{\mathrm{M}_{<X}}{\mathrm{M}_{\mathrm{T}}}=\left(\frac{\mathrm{X}}{\mathrm{X}_{\max }}\right)^{3-\mathrm{D}_{\mathrm{t}}}$

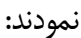

كه X

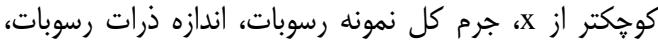

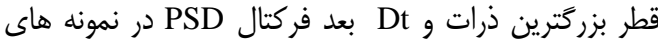

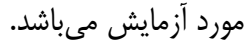

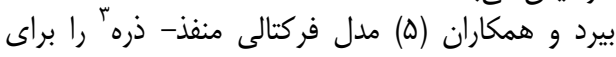

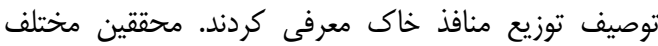

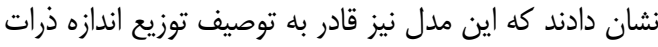

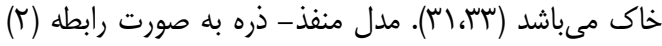

$\mathrm{M}\left(\mathrm{X} \leq \mathrm{X}_{\mathrm{i}}\right)=\mathrm{C}_{\mathrm{b}} \mathrm{X}_{\mathrm{i}}^{3-\mathrm{D}_{\mathrm{b}}}$ مىباشد.

جرم تجمعى ذرات كوخگتر از X (X SXi)

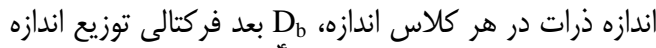

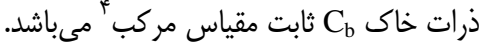

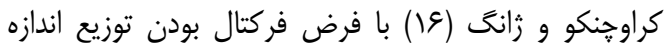

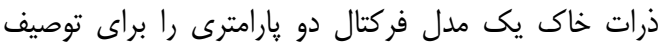
توزيع اندازه ذرات خاك ارائه كردند.

$M(X)=\exp \left\{\ln C_{K}+\left[\frac{3 D^{2}-13 D+14}{D^{2}-5 D+4} 1\right] \ln X\right\}$

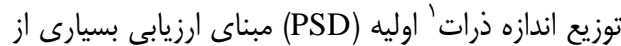

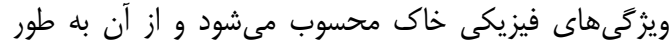

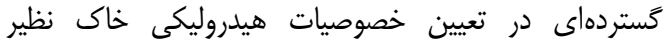
منحنى رطوبتى، و هدايت آبى اشباع و غير غير اشباع استفادها

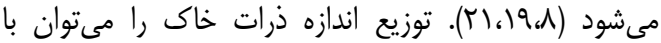

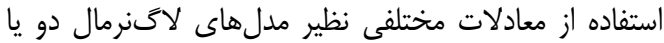

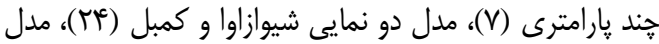

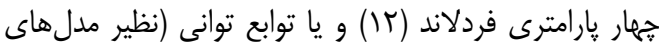

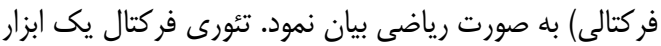

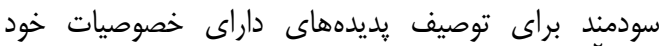

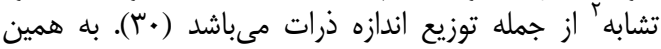

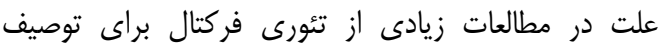

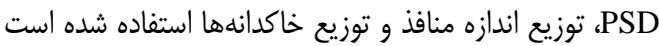

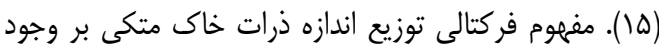

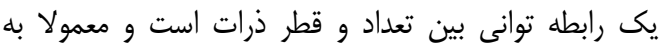

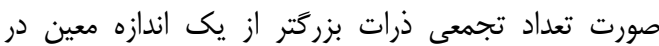

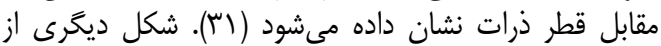

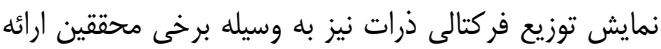

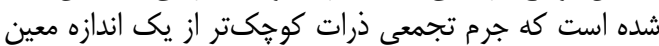

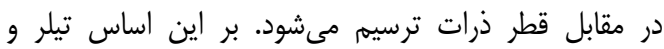

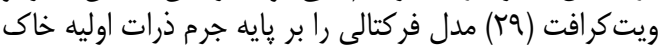

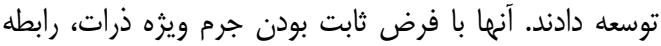

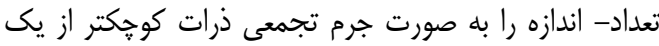

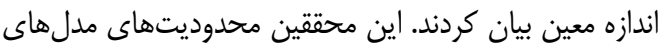
مبتنى بر تعداد- اندازه را نيز مورد بردسى مجنى قرار داده و نشان مدان مدان 
توزيع اندازه ذرات خاك را براى توصيف PSD رسوبات در

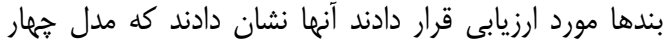

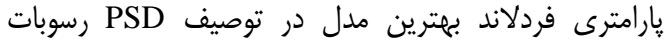

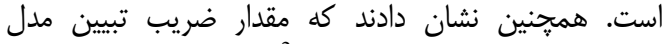

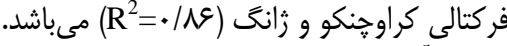

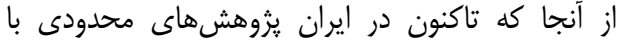

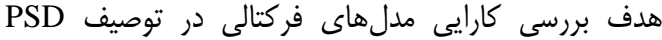

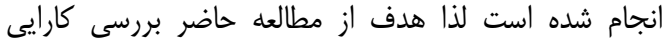

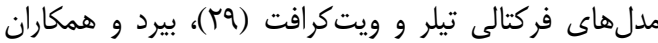

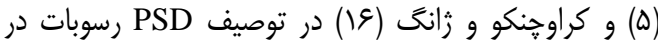

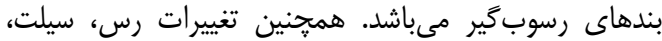

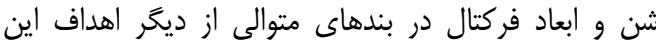

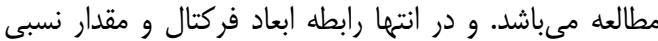

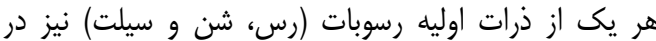
PSD رسوبات مورد مطالعه قرار مى گيرد.

\section{مواد و روشها} موقعيت منطقه مورد يزوهش رونش

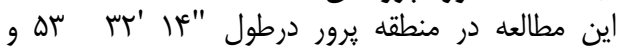

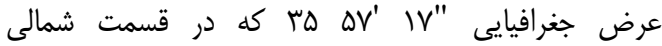

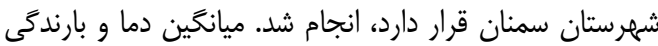

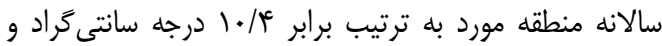

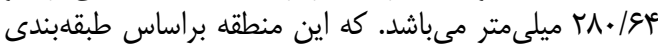

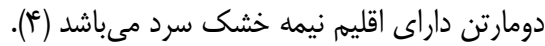

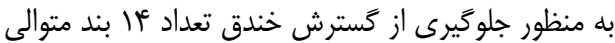

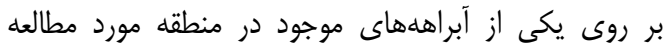

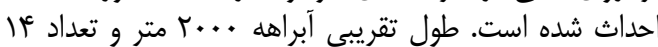

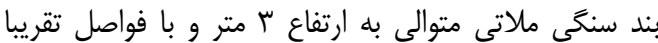

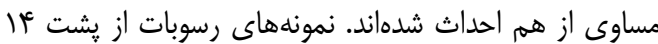

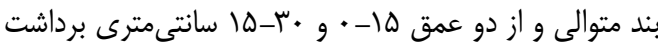

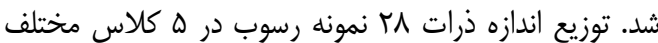

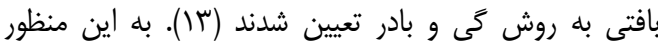

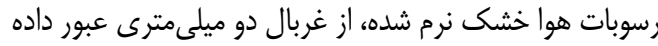

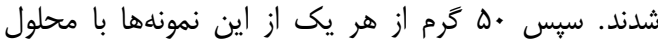

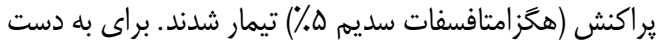

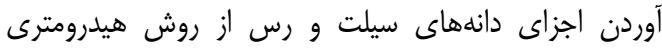

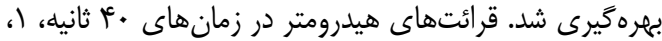

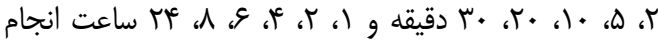

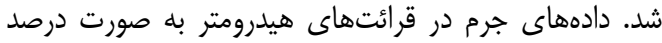

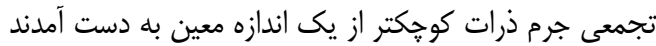

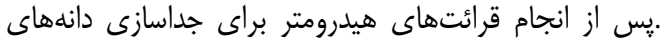

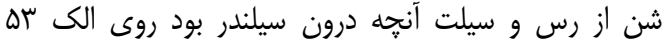

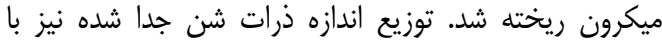

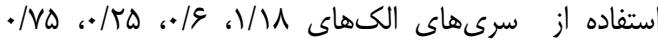
ميلى متر تعيين شد.

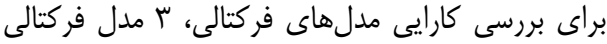

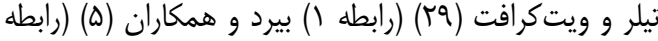

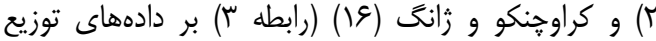
اندازه ذرات رسوب برازش داده شدند. برازش مدلها ازئ طان طريق
كه D بعد فر كتالى توزيع اندازه ذرات خاك و Ck ضريب ثابت

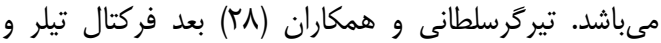

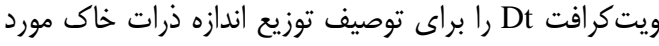

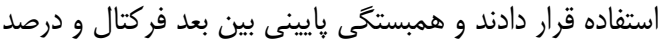
شن، رس و سيلت خاك مشاهده نمودند. زهاو و ور همكاران

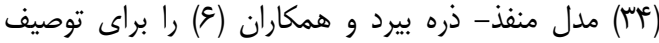

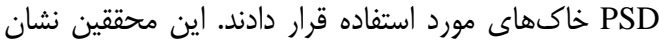

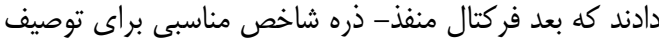

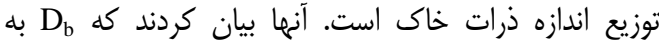

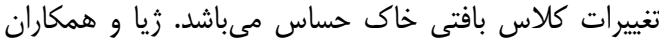

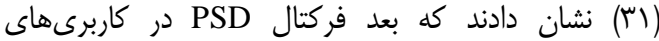

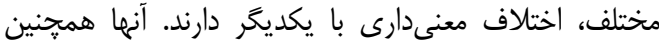

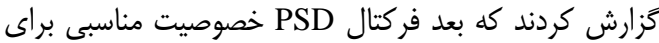

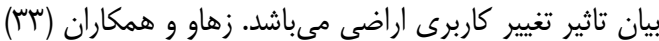

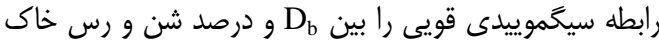

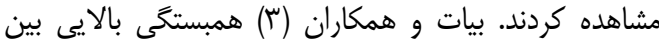

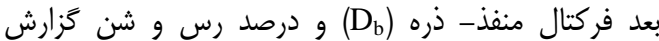

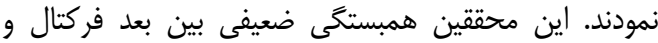

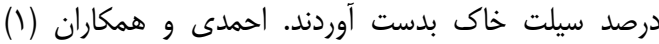

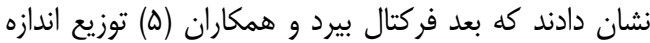

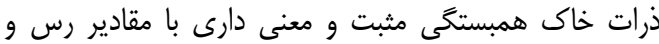

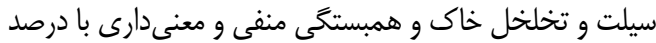

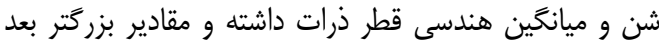

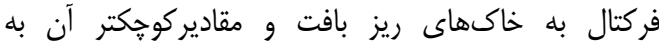
خاكهاى درشت بافت تعلق داشته است. در مطالعهى ديخرى

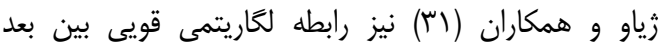

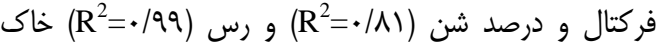

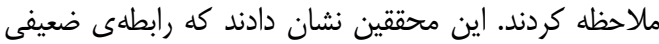

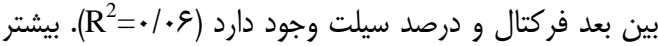

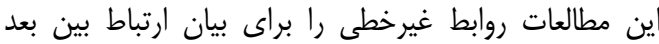

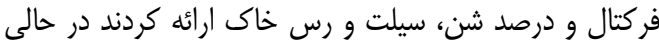

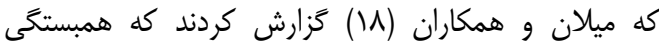
خطى معنى دارى بين بعد فركتال و درصد رس خار خاى وجود

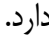

اهميت بررسى توزيع اندازه ذرات رسوبات در بندهاى

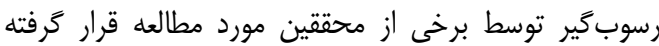

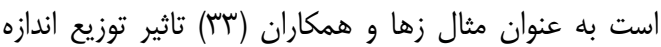

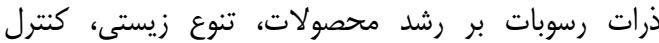

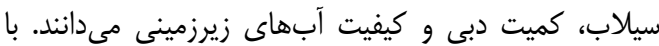

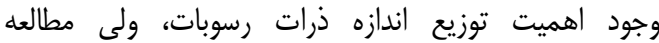

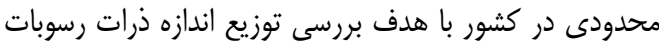

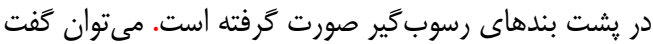

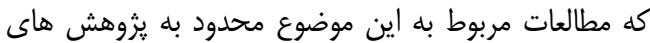

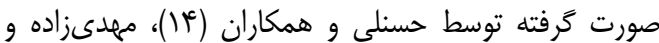

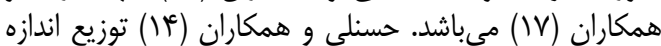

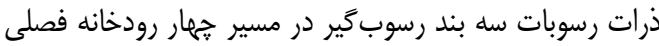

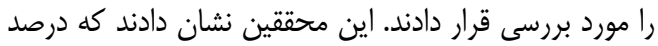

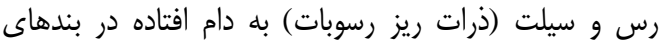

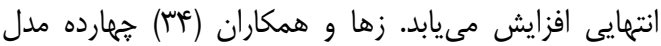


كه در آن AICc، معيار اطلاعات آكايك اصلاح شده، MSE

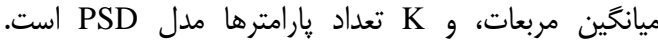
ميانكين مربعات با استفاده از معادله زير تعيين شدار مئ

MSE $=\frac{\sum_{i=1}^{N}\left(\mathrm{Y}_{\mathrm{i}}-\mathrm{Y}_{\mathrm{p}}\right)^{2}}{\mathrm{~N}}$

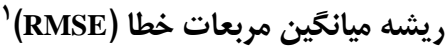

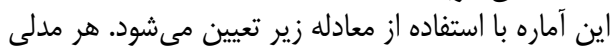

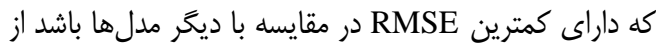
دقت بيشترى برخوردار است.

$$
\mathrm{RMSE}=\sqrt{\frac{\sum_{\mathrm{i}=0}^{\mathrm{N}}\left(\mathrm{Y}_{\mathrm{i}}-\mathrm{Y}_{\mathrm{p}}\right)^{2}}{\mathrm{~N}}}
$$

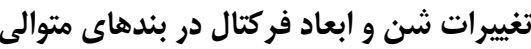

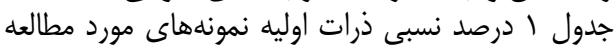

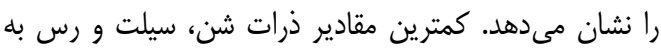

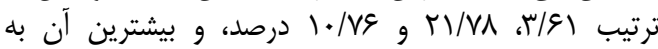

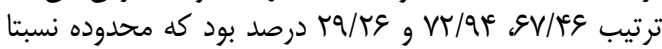

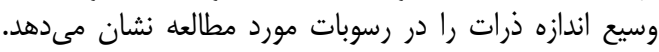

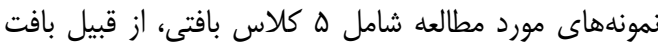

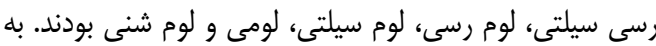

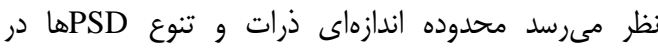

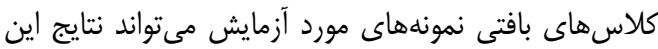

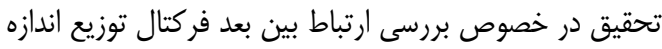

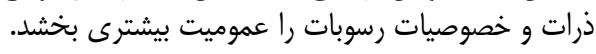

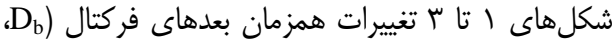

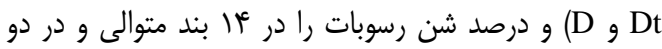

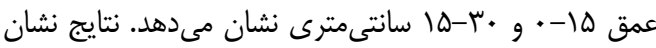

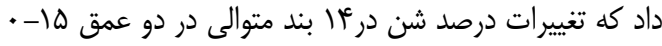

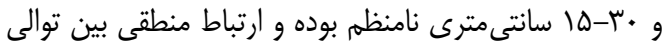

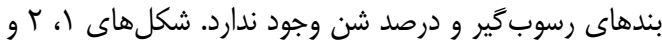

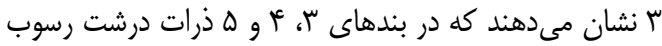

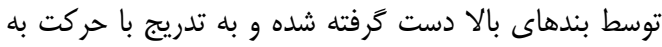

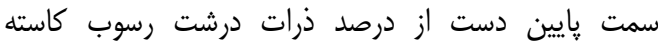

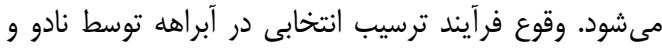

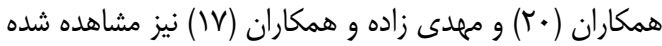

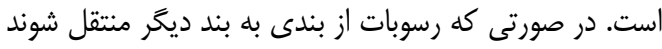

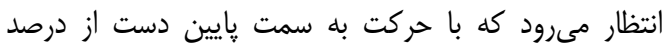

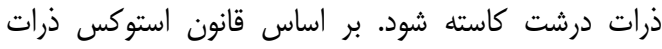

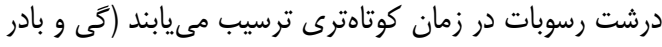

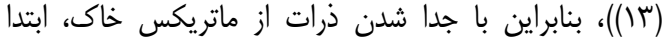

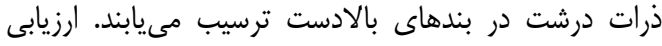

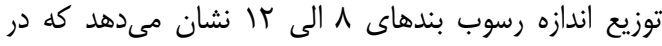

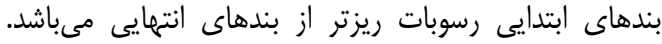

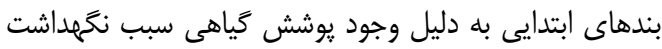

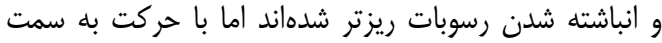

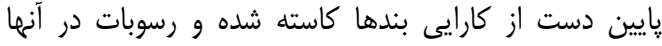

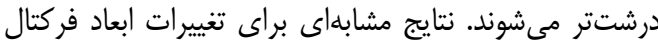

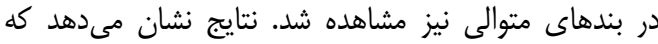

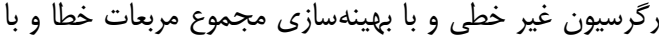

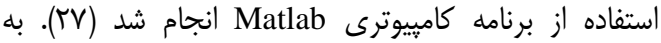

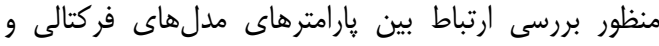

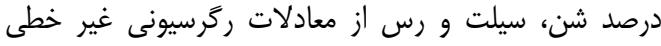

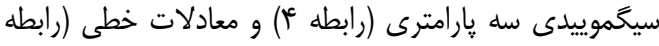

$$
\begin{aligned}
& \mathrm{y}=\frac{a}{1+\mathrm{e}^{-\left(\frac{\mathrm{x}-\mathrm{x}_{0}}{\mathrm{~b}}\right)}}
\end{aligned}
$$

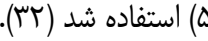

$$
\begin{aligned}
& \mathrm{y}=a_{2} \mathrm{x}+b_{2}
\end{aligned}
$$

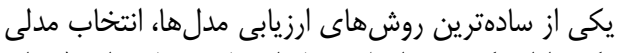

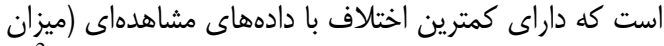

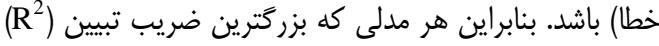

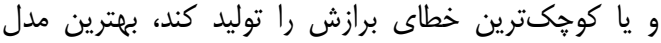

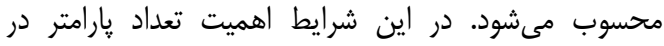

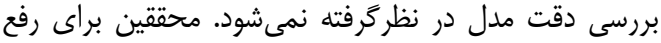

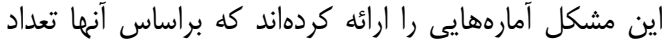

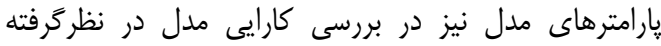

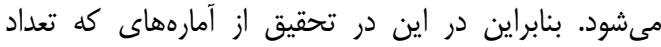

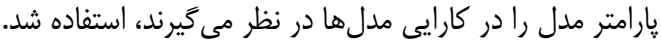

اين آمارهها عبارتند از:

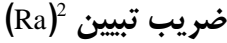

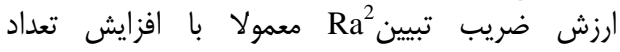

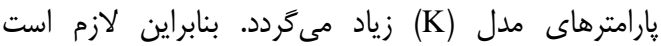

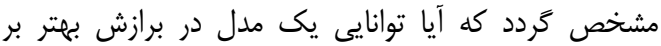

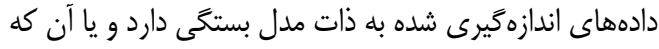

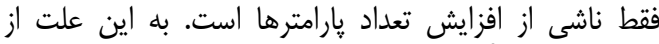

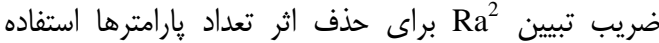

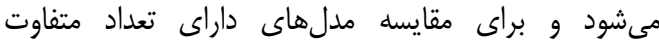
يارامترها، مورد استفاده قرار مى گيرد (سائ).

$\mathrm{R} a^{2}=1-\left(\frac{\mathrm{N}-1}{\mathrm{~N}-\mathrm{K}}\right) \frac{\mathrm{SSE}}{\mathrm{SST}}$

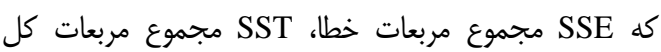

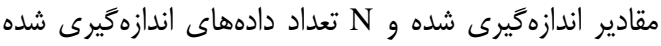

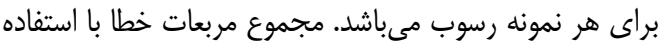
از معادله زير تعيين شد.

$\operatorname{SSE}=\sum_{\mathrm{i}=0}^{\mathrm{N}}\left(\mathrm{Y}_{\mathrm{i}}-\mathrm{Y}_{\mathrm{p}}\right)^{2}$

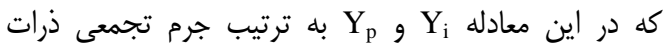

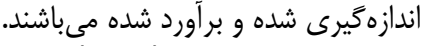
معيار اطلاعات اكايك (AICc) اصلاح شده شاره

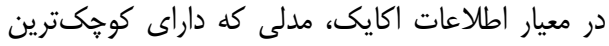

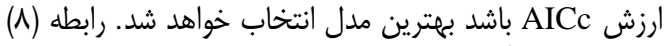
نحوه محاسبه آماره اكايك را نشان مىدهد (A)

$\mathrm{AIC}_{\mathrm{c}}=\mathrm{N} \times \ln (\mathrm{MSE})+2 \times \mathrm{K}+\frac{2 \mathrm{~K}(\mathrm{~K}+1)}{\mathrm{N}-\mathrm{K}-1}$ 
رسوبات در بندهاى پايين دست، ريزتر از خاك اصلى مىباشد.

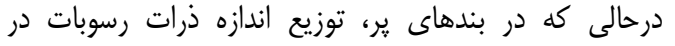

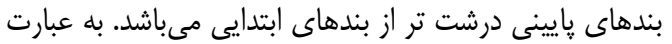

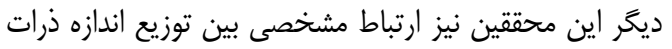
رسوبات و توالى بندها مشاهده نكردند.

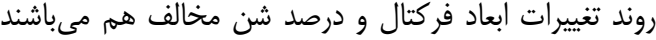

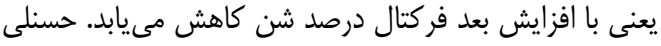

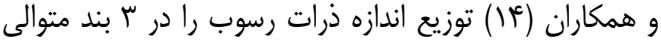

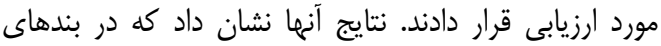

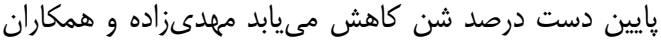

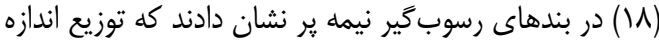

Table 1. Percentage of particle sediment in study area

جدول ا- درصد نسبى ذرات اوليه رسوبات مورد مطالعه

\begin{tabular}{|c|c|c|c|c|c|c|}
\hline Dt & D & $\mathrm{Db}$ & درصد سيلت & درصد رس & درصد شن & خصوصيت \\
\hline$r / q$. & $r / A V$ & $r / 9 \Lambda$ & $r / / v \Lambda$ & $1 . / \mathrm{VY}$ & $\Gamma / 91$ & حداقل \\
\hline$r / 99$ & $r / 90$ & T/AV & $V Y / q T$ & rq/रq & $9 V / 49$ & حداكثر \\
\hline$r / \Lambda \mu$ & س T/q & $r / \Lambda$. & $|f \Delta / F|$ & $r r / 19$ & $r r / r q$ & متوسط \\
\hline
\end{tabular}

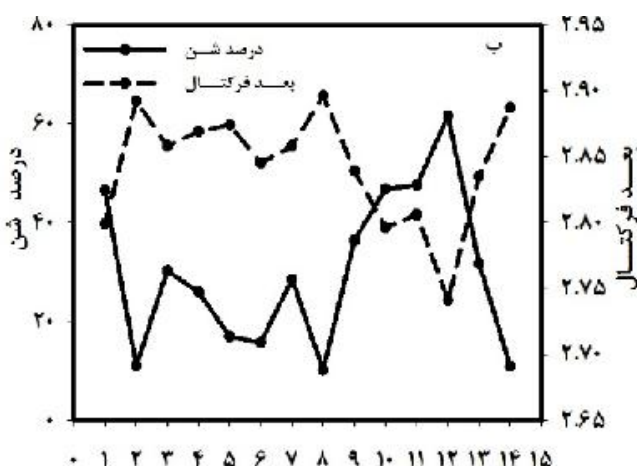

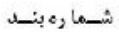

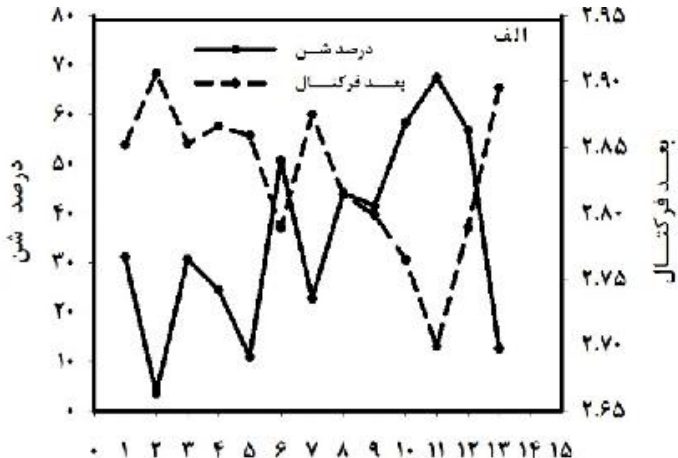

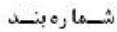

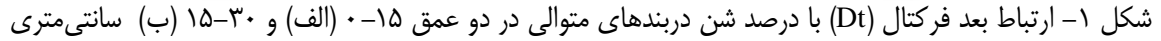

Figure 1. The relationship between fractal dimension (Dt) and sand content in successive check dams in depths of 015 and $15-30 \mathrm{~cm}$

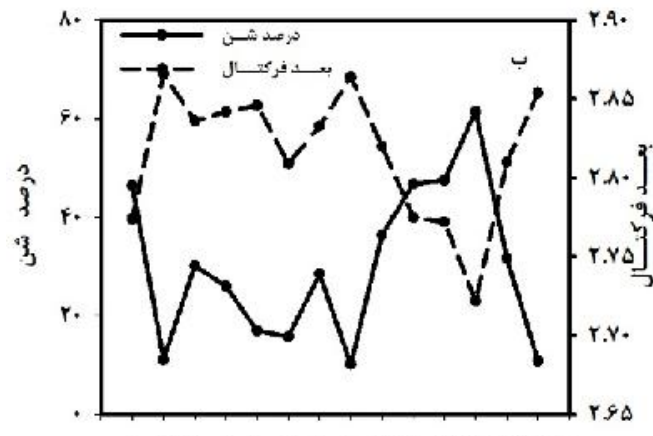

شـماره بنــ

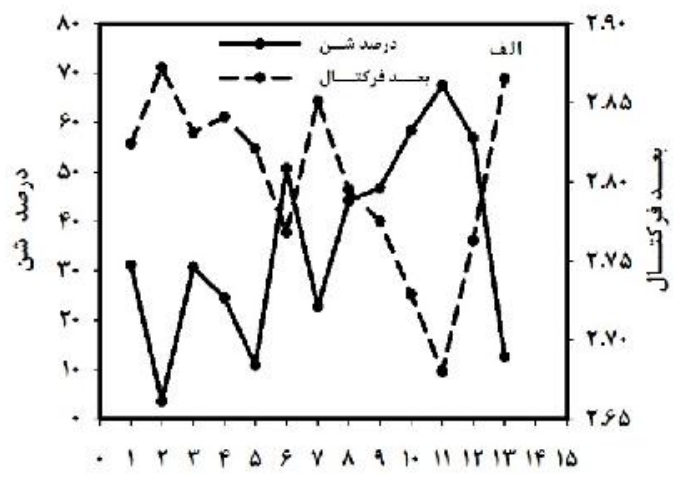

شــــاره بنــن

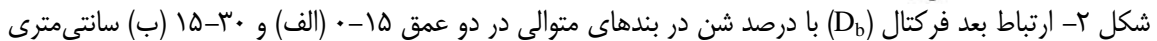

Figure 2. The relationship between fractal dimension $\left(\mathrm{D}_{\mathrm{b}}\right)$ and sand content in successive check dams in depths of $0-15$ and $15-30 \mathrm{~cm}$ 

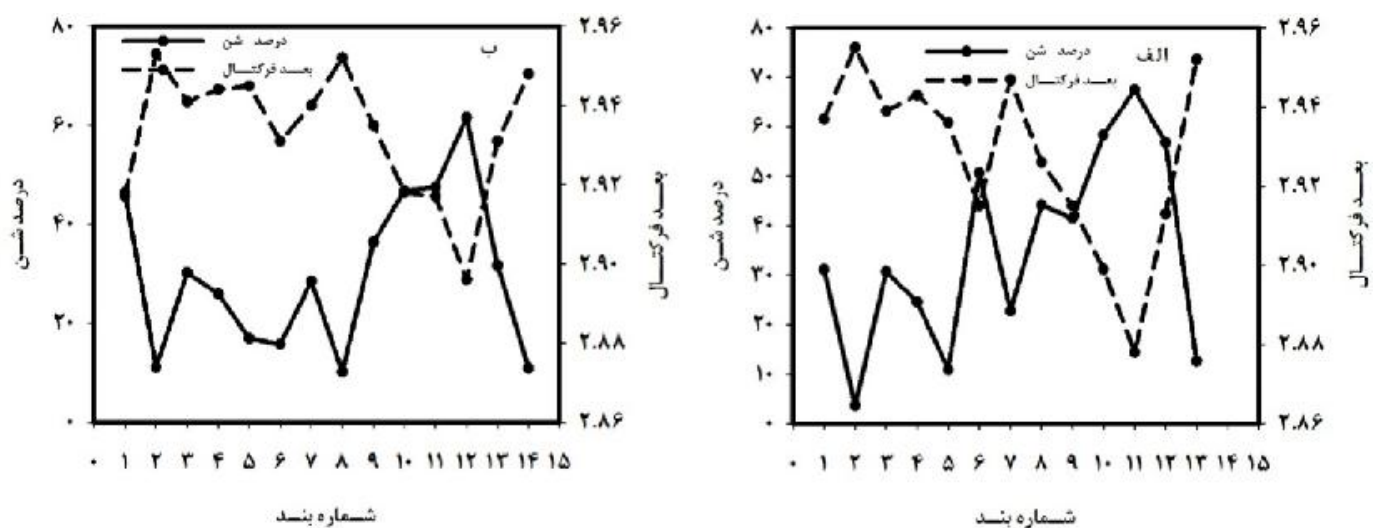

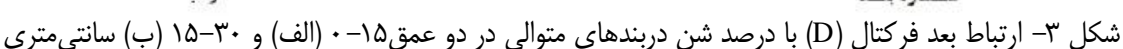

Figure 3. The relationship between fractal dimension (D) and sand content in successive check dams in depths of 015 and $15-30 \mathrm{~cm}$

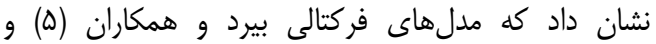

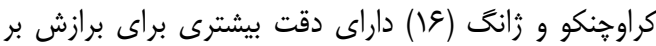

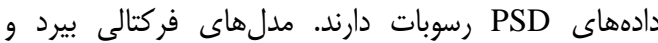

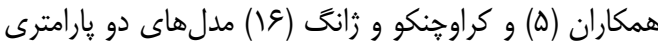

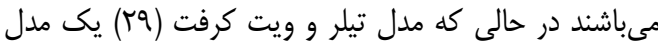

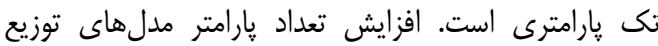

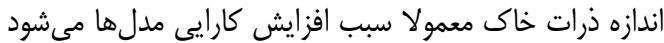

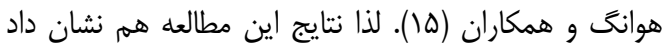

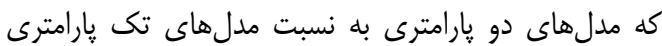

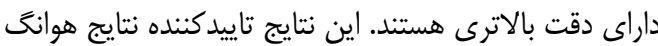

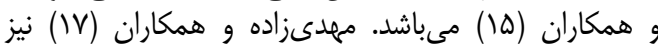

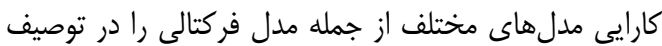

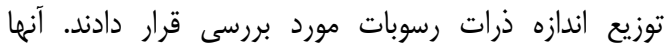

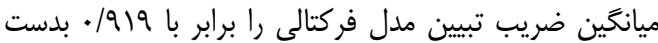

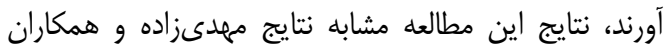

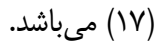

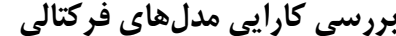

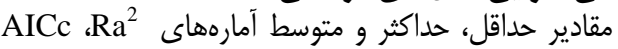

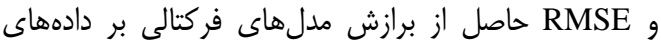

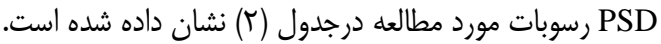

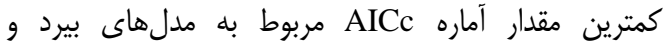

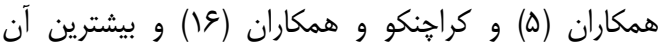

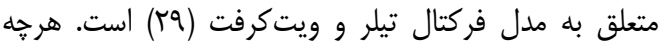

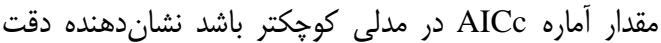

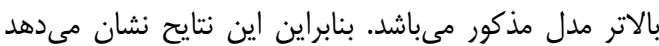

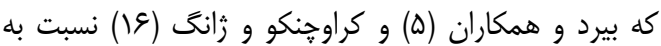

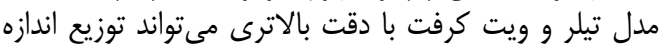

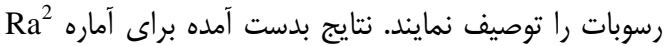

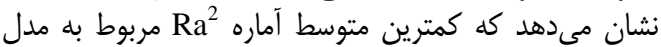

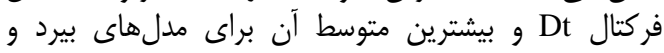

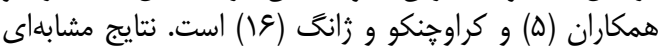
براى آماره RMSE نيز بدست آمد. بهطور كلى تحليل آمارهها

Table 2. Statistics criteria of AICc،Ra ${ }^{2}$ and RMSE in fractal models

\begin{tabular}{|c|c|c|c|c|c|c|c|c|c|}
\hline $\begin{array}{r}\text { متوسط } \\
\text { RMSE }\end{array}$ & $\begin{array}{l}\text { حداكثر } \\
\text { RMSE }\end{array}$ & $\begin{array}{l}\text { حداقل } \\
\text { RMSE } \\
\end{array}$ & Ra² متوسط & $\begin{array}{l}\text { حداكثر } \\
\mathrm{Ra}^{2}\end{array}$ & $\begin{array}{l}\text { حداقل } \\
\mathrm{Ra}^{2}\end{array}$ & $\begin{array}{l}\text { متوسط } \\
\text { AICc }\end{array}$ & $\begin{array}{l}\text { حداكثر } \\
\text { AICc }\end{array}$ & حداقل & فركتال ها \\
\hline$\cdot / 1$ & $\cdot / 19$ & $\cdot / \cdot f$ & $\cdot / \wedge \mu$ & $\cdot / 9 \mathrm{~V}$ & $\cdot / \mathrm{QV}$ & $-M Y / T I$ & $-9 / T 8$ & $-\Delta V / \& q$ & تيلروويت كرفت \\
\hline .1 .9 &.$/ \mathrm{V}$ & $.1 \cdot 4$ & $\cdot / \Lambda \Delta$ &.$/ 97$ &.$|9|$ & -זه/q & $-|1 / 4|$ & TET/KT & بيردوهمكاران \\
\hline
\end{tabular}

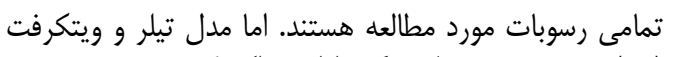

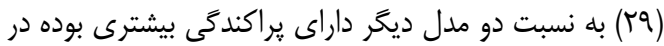

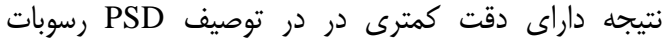

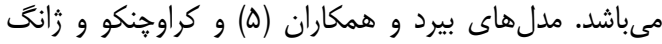

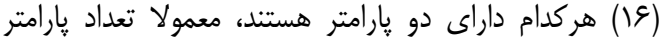

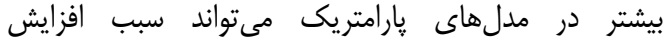

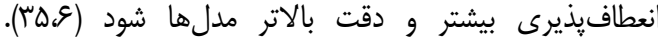

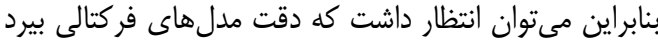

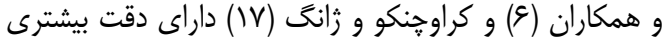
نسبت به مدل تيلر و ويتكرفت باشند.

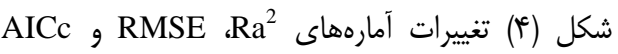

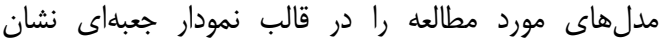

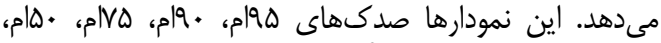

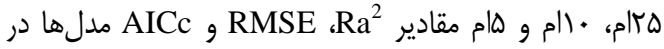

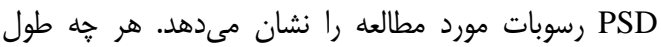

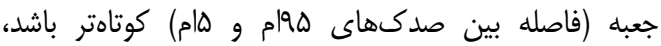

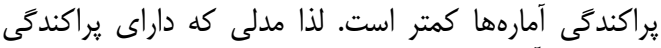

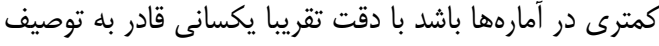

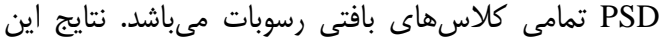

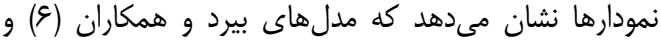

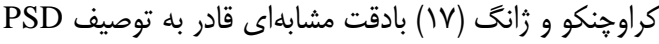




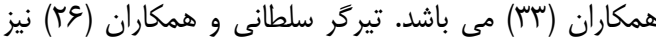

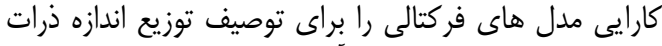

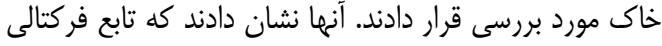

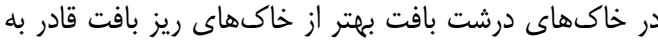
توصيف PSD مىباشد.
زها و همكاران (بّآ) مدل بيرد و همكاران (ه) را براى توصيف

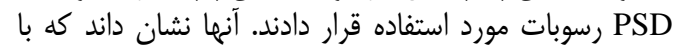

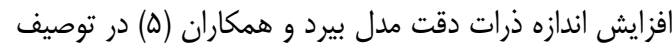

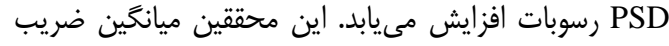

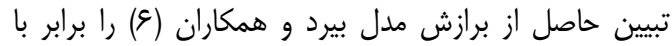

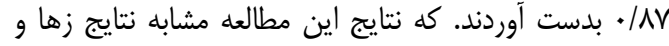
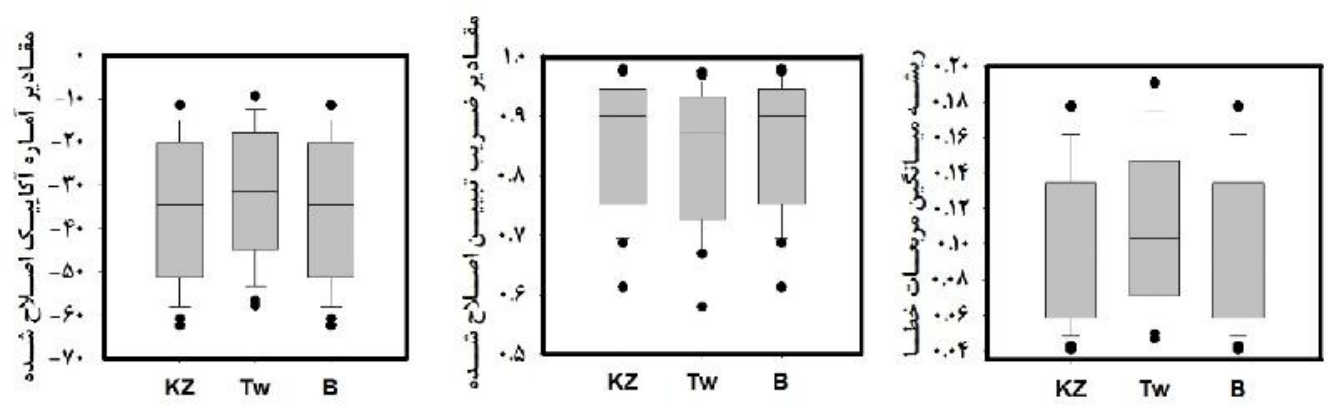

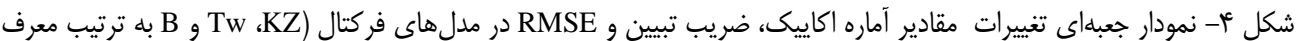

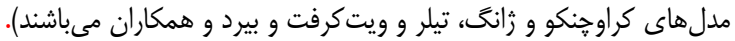

Figure 4. The box plot of distribution of AICc $\mathrm{Ra}^{2}$ and RMSE criteria in different fractal models. (KZ, Tw and B refer to Kravchenko and Zhang, Tyler and Wheatcraft and Bird fractal models, respectively)

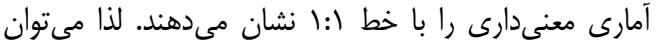

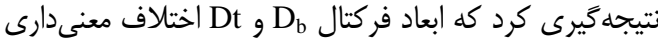

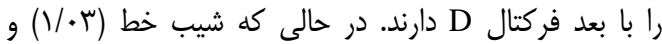

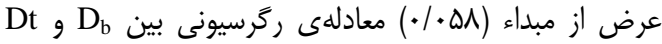

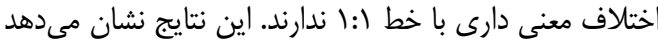

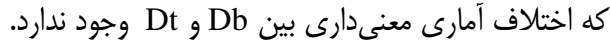

بر رسى ارتباط بين ابعاد فركتال

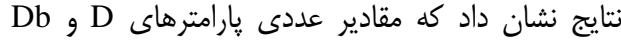

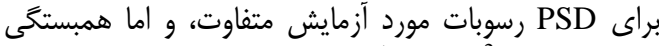

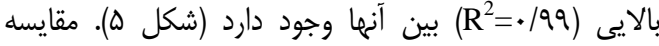

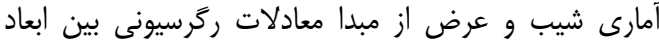

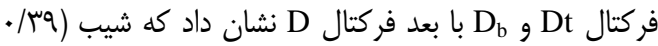

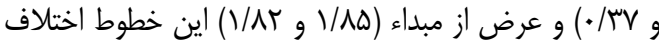
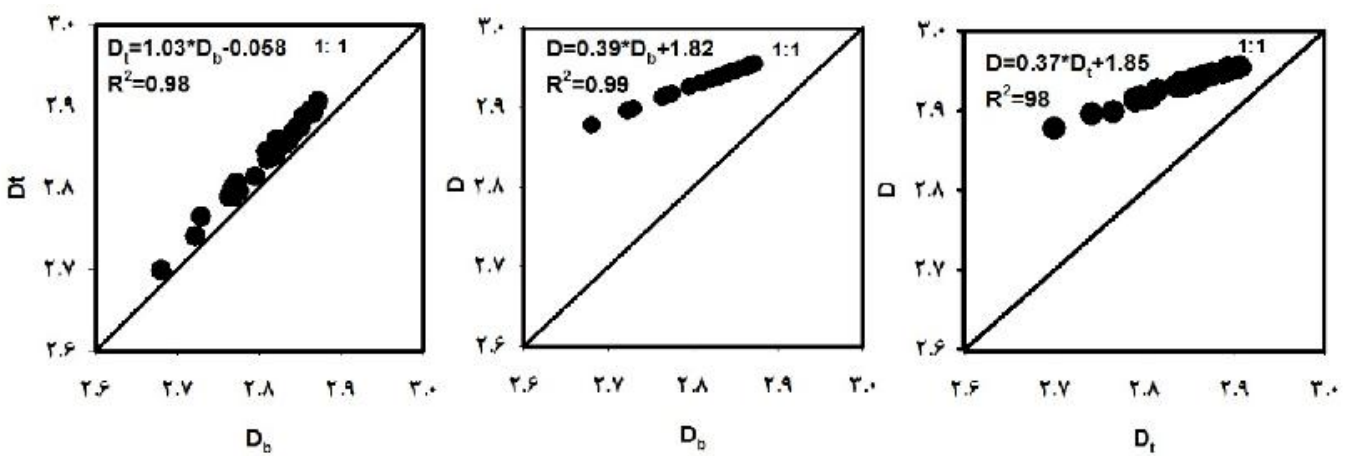

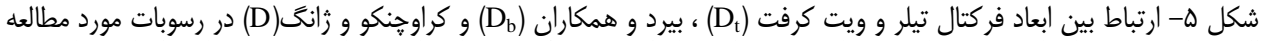

Figure 5. The relation between Tyler and Wheatcraft $\left(D_{t}\right)$, Bird et al $\left(D_{b}\right)$ and Kravchenko and Zhang (D) in sediment in study area

اشارهاى نكردند. ارساهين و همكاران (•(1)، و سو و همكاران

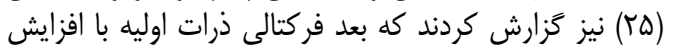

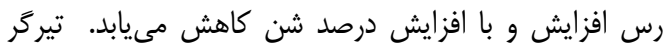

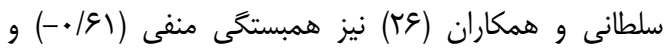

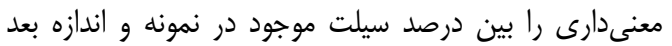

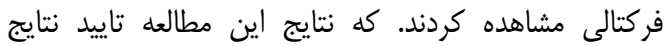

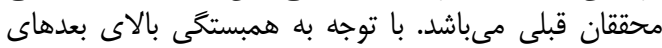

بررسى ارتباط بين ابعاد فركتال و پارامترهاى توزيع

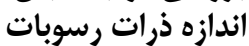

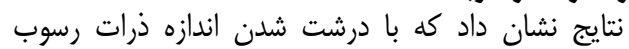

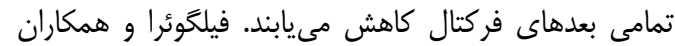

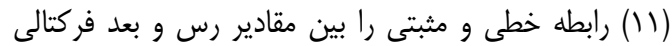

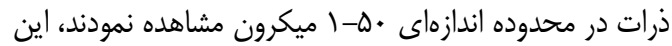

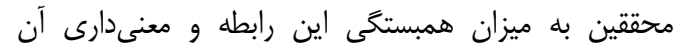




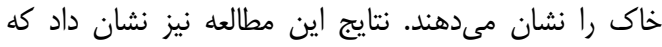

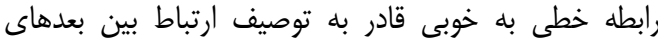

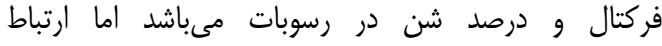

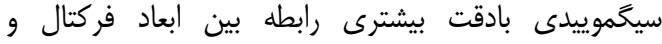

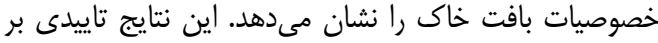

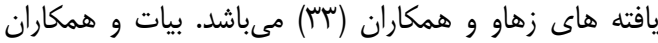

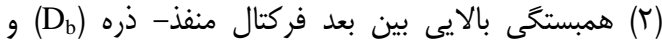

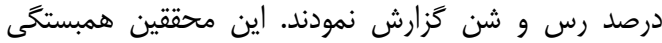

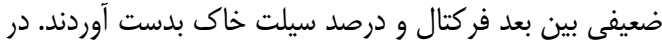

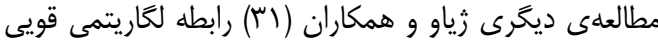

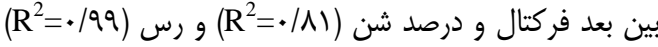

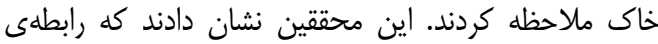

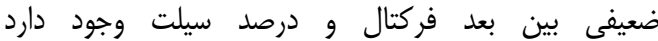

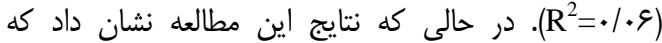

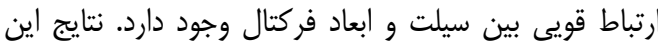

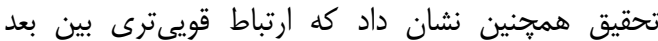

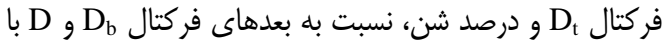

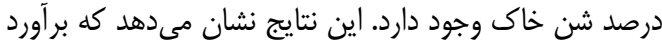
با باستفاده از مقادير شن به خوبى امكان يذير مى باشد.
فر كتال با درصد شن، سيلت و رس مت توان از بعدهاى فركتال

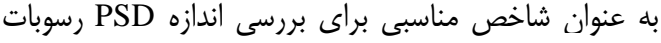

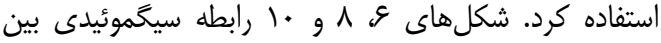

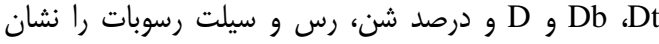

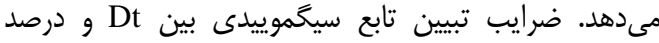

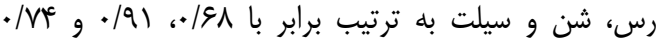

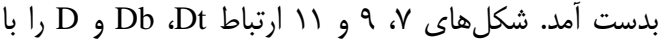

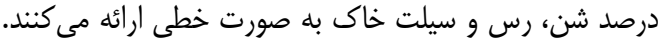

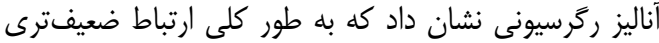

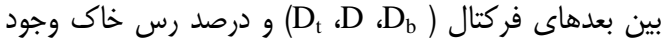

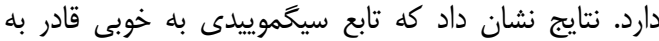

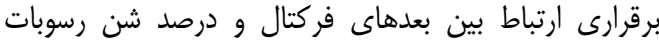

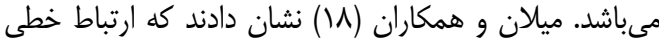

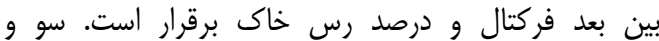

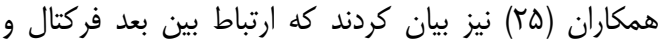

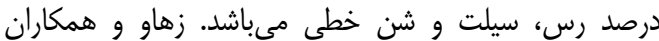

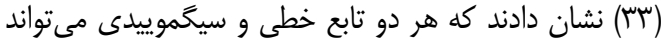

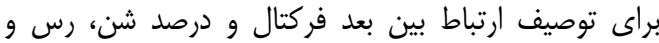

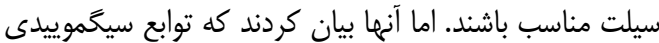
با دقت بالاترى رابطه بين ابعاد فركتال و خصوصيات بان بافت بان

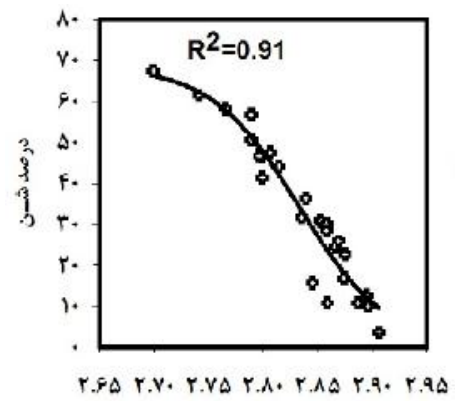

$D_{t}$

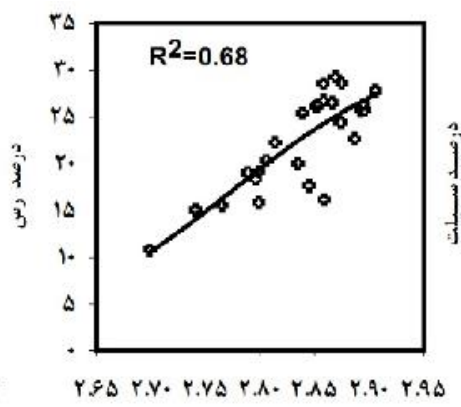

$D_{t}$

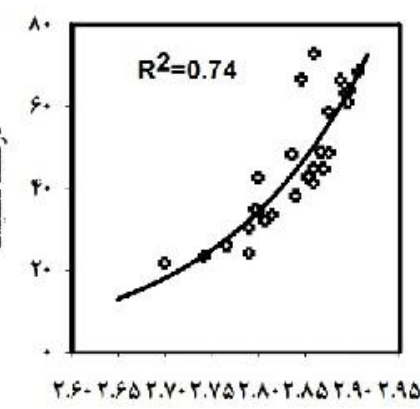

$D_{t}$

شكل צ- رابطه بين Dt و درصد شن، رس و سيلت خاى (Rt نشاندهنده ضريب تبيين برازش معادله سيخموييدى بر دادههاى اندازمكيرى شده مىباشد).

Figure 6. The relation between sand, silt and clay and $D_{t}\left(R^{2}\right.$ is coefficient of determination of sigmoid equation that fitting on measurement data)

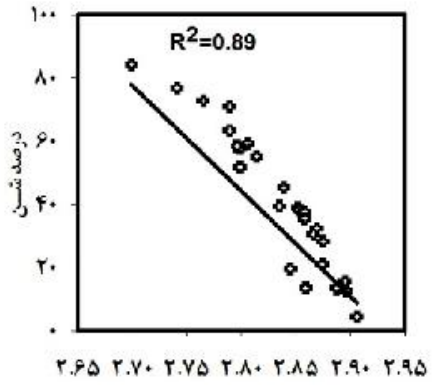

$D_{t}$

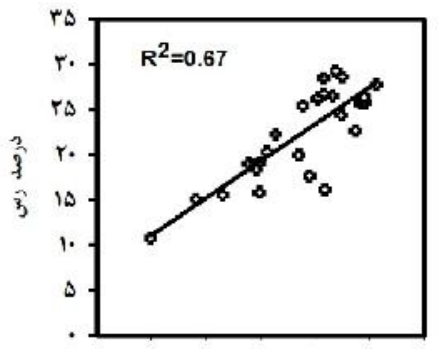

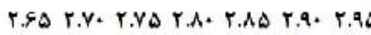

$D_{t}$

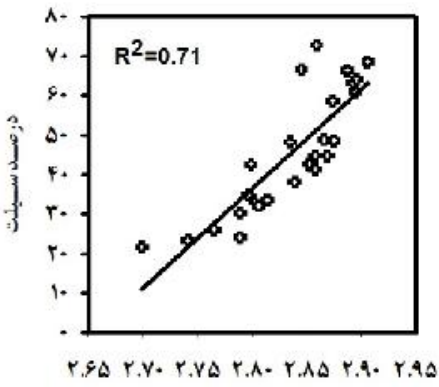

$D_{\mathbf{t}}$

شكل V- رابطه بين Dt و درصد شن، رس و سيلت خاك (Rt نشاندهنده ضريب تبيين برازش معادله خطى بر دادهاى اندازمخيرى شده مىباشد).

Figure 7. The relation between sand, silt and clay and $D_{t}\left(R^{2}\right.$ is coefficient of determination of linear equation that fitting on measurement data) 


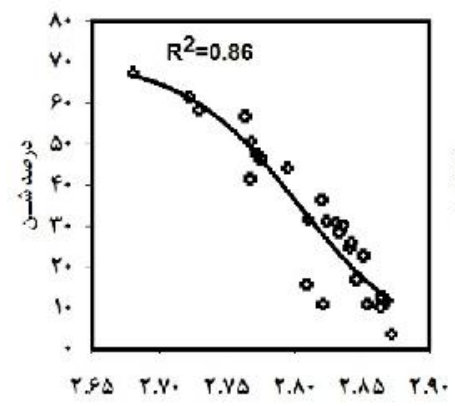

$\mathbf{D}_{\mathbf{b}}$

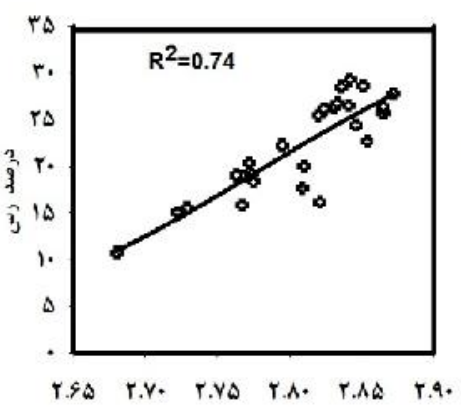

$\mathrm{D}_{\mathrm{b}}$

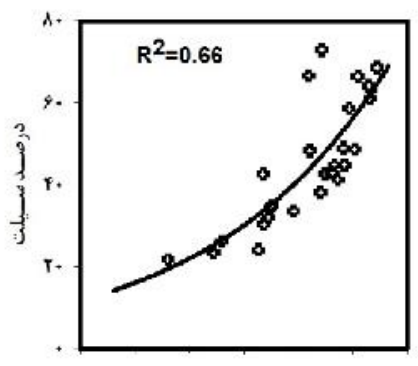

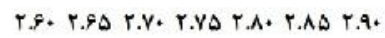

$D_{b}$

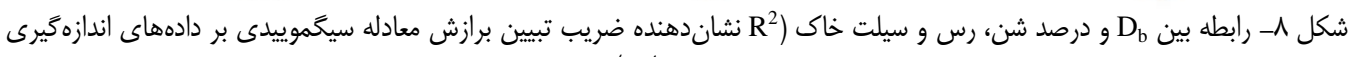
شده مىباشد).

Figure 8. The relation between sand, silt and clay and $\mathrm{D}_{\mathrm{b}}\left(\mathrm{R}^{2}\right.$ is coefficient of determination of sigmoid equation that fitting on measurement data)

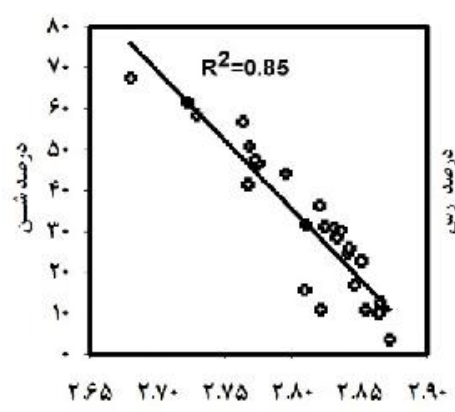

$\mathrm{D}_{\mathrm{b}}$

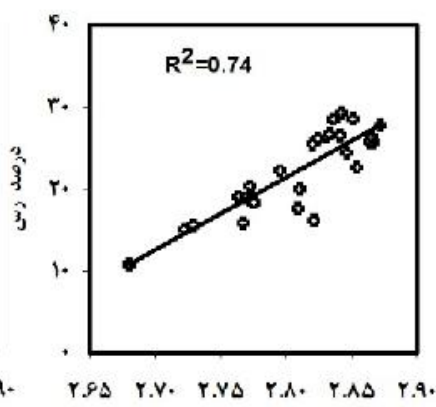

$\mathrm{D}_{\mathrm{b}}$

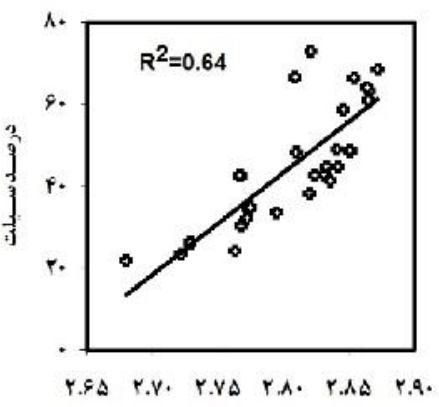

$D_{\mathbf{b}}$

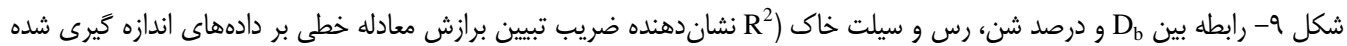
مى باشد).

Figure 9. The relation between sand, silt and clay and $\mathrm{D}_{b}\left(\mathrm{R}^{2}\right.$ is coefficient of determination of linear equation that fitting on measurement data)

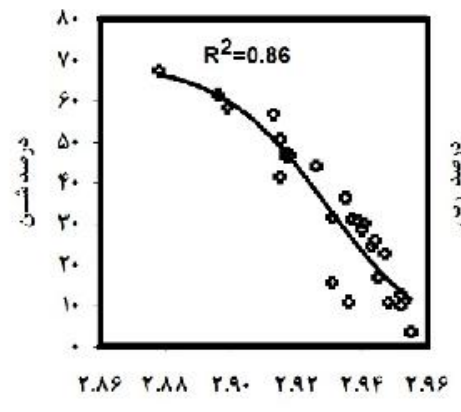

D

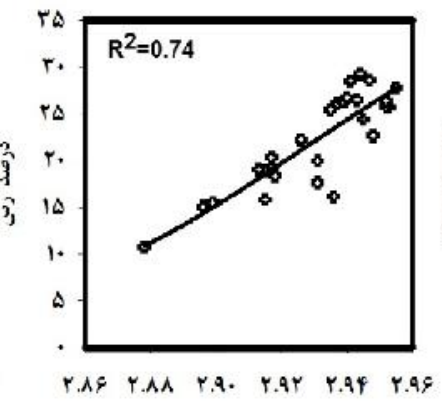

D

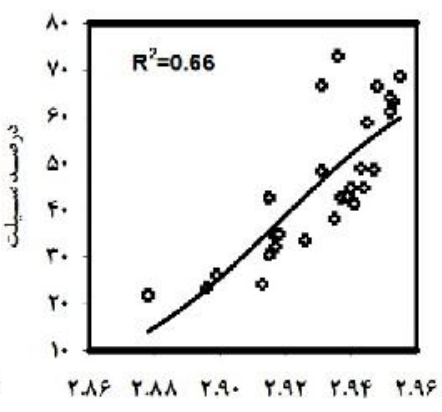

D

شكل • ا - رابطه بين D و درصد شن، رس و سيلت خاك (R نشان دهنده ضريب تبيين برازش معادله سيخموييدى بر دادههاى اندازهخيرى شده مىباشد).

Figure 10. The relation between sand, silt and clay and $\mathrm{D}\left(\mathrm{R}^{2}\right.$ is coefficient of determination of sigmoid equation that fitting on measurement data) 


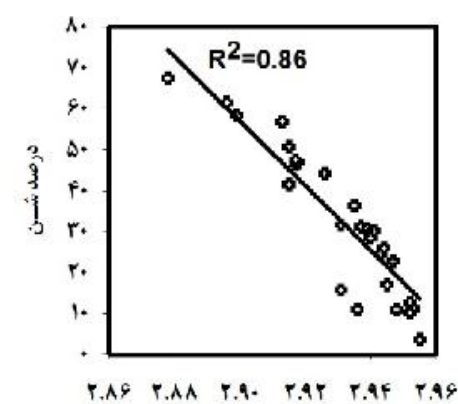

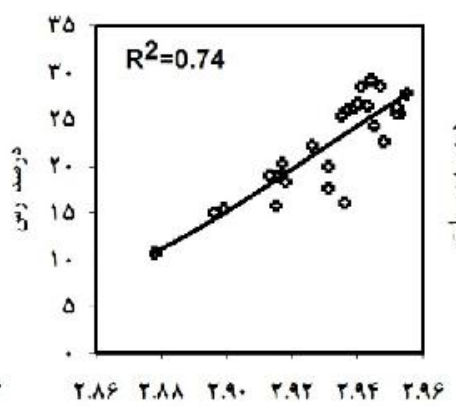

D

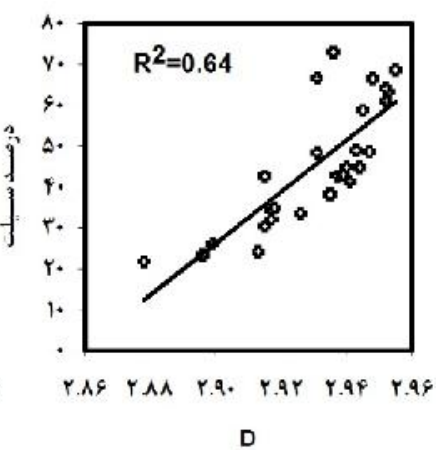

D

شكل لI - رابطه بين D و درصد شن، رس و سيلت خاى (R2 نشان دهنده ضريب تبيين برازش معادله خطى بر دادهاى اندازهگيرى شده مىباشد).

Figure 11. The relation between sand, silt and clay and $\mathrm{D}\left(\mathrm{R}^{2}\right.$ is coefficient of determination of linear equation that fitting on measurement data)

فركتالى كاهش مى يابد. ارتباط بين دقت برازش مدلهاى

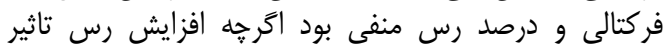

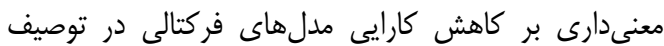

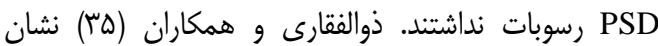
دادند كه توزيع اندازه ذرات خاك بات با ريزتر شدات فيدان اندازه ذرات

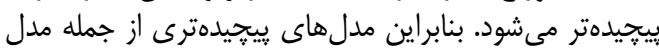

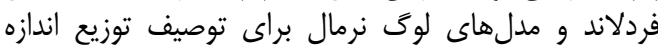

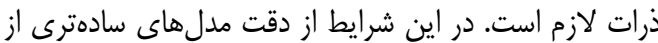
قبيل مدل فركتال كاسته مى شود.
همبستخى بين خطاى برازش (RMSE) حاصل از برازش

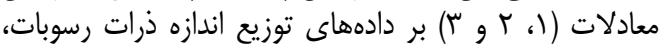

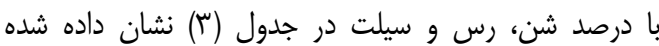

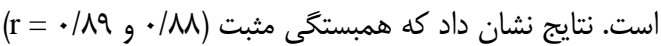

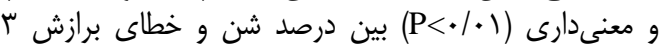

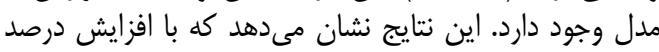

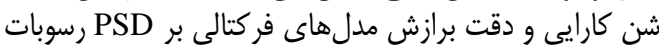

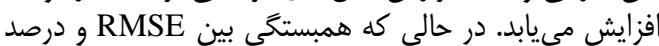

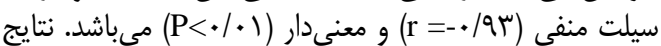
نشان مىدهد كه با افزايش سيلت دقت برازش مدلهاي مياى

جدول ب- همبستخى (r) بين دقت برازش (RMSE)مدلهاى فركتالى و درصدشن، رس و سيلت رسوبات Table 3. Correlation coefficient (r) of between accuracy of fitting (RMSE) in fractal models and sand, silt and clay content in sediment

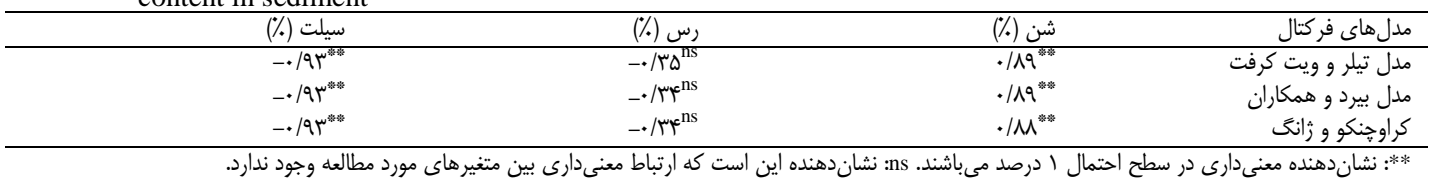

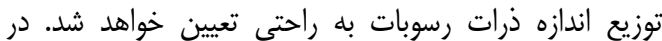

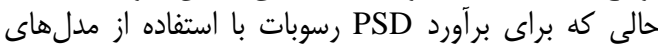

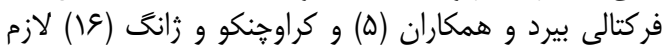

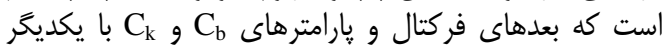

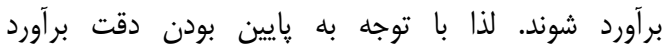

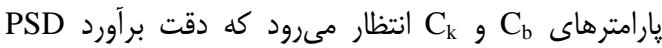

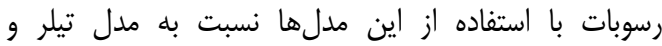

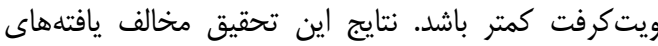

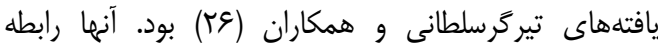

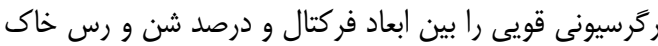

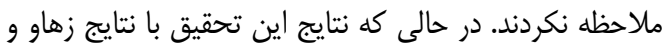

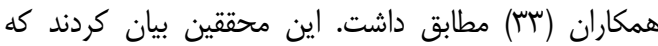
ارتباط قويى بين ابعاد فركتال در درصد رس، دران و شن رسون رسوبات

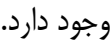

به منظور تعيين و توصيف ارتباط بين بعدهاى فركتال و ماك

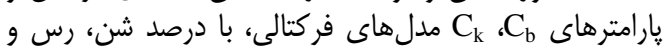

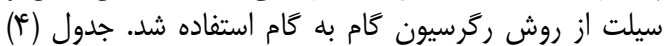

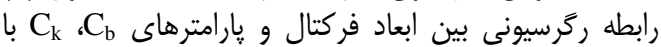

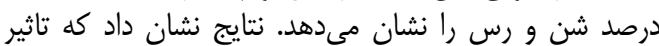

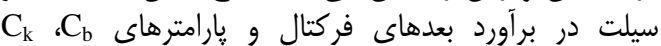

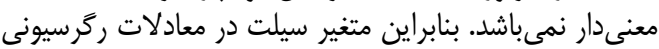

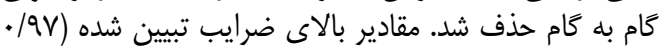

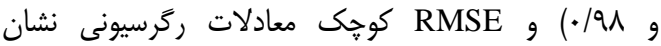

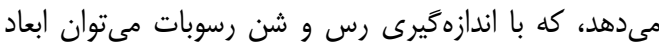

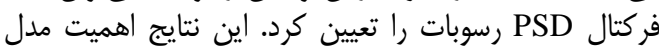

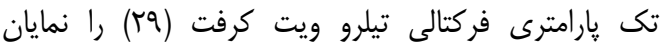

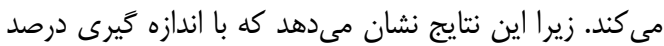

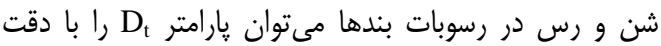

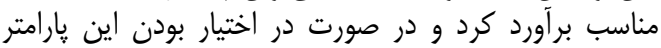


جدول fا- روابط ركرسيونى بين ابعاد فركتال و نسبت شن، رس در PSD رسوبات Table 4. The multiple linear regression between fractal dimension and sand and clay content in sediment

\begin{tabular}{|c|c|c|}
\hline$D_{t}=-r / \wedge \backslash \wedge \times$ Sand $+\cdot / r v r \times$ clay $+r / \wedge ı \wedge$ & $\mathrm{R}^{r}=\cdot / 9 \mathrm{~V}$ & RMSE $=\cdot / \cdot \bullet \wedge \wedge$ \\
\hline $\mathrm{Db}=-\cdot / \mathrm{I} \vee r q \times$ Sand $+\cdot / \mu q v \times$ clay $+r / v q \varepsilon$ & $\mathrm{R}^{r}=\cdot / 91$ & RMSE $=\cdot / \cdot V^{\epsilon}$ \\
\hline $\mathrm{D}=-. / \cdot \vee_{\wedge} r \times$ Sand $+. / / \vee \Delta \times$ clay $+r / १ / r$ & $\mathrm{R}^{r}=\cdot / 9 \mathrm{~V}$ & RMSE $=. / . . r \mu$ \\
\hline $\mathrm{Cb}=-\cdot / \mu \cdot \Delta 1 \times$ Sand $+\cdot / \cdot r \cdot \times$ clay $+1 / 1$. & $\mathrm{R}^{r}=\cdot / 9$ & RMSE $=\cdot / \cdot 1 \wedge$ \\
\hline $\mathrm{C} k=-\cdot /$ r $\Lambda \times$ Sand $-\cdot / \cdot r \cdot r \times$ clay $+1 / \cdot q$ & $\mathrm{R}^{r}=\cdot / q$. & RMSE $=\cdot / \cdot 1 \mathrm{~V}$ \\
\hline
\end{tabular}

زانگ (ع) رانط رات توصيف نمايند. نتايج اين مطالعه نشان داد كه

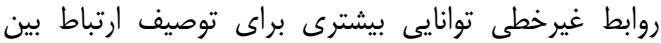

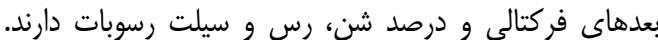

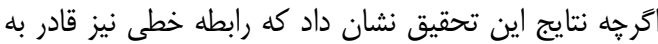

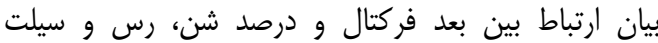

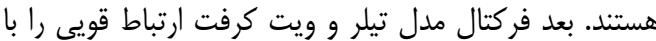

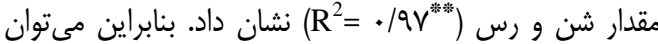

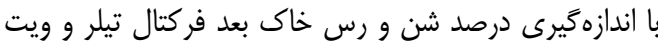

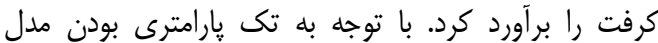

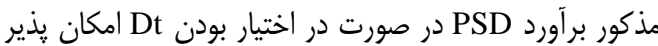

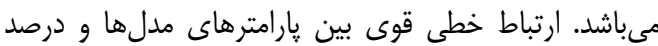

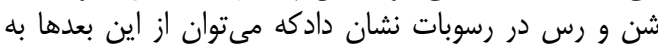

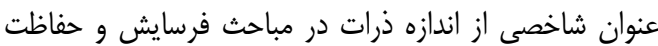

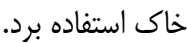

برازش مدلهاى تيلر و ويتكرافت (وج)، بيرد و همكاران

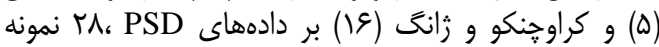

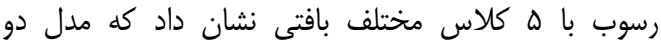

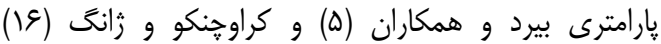

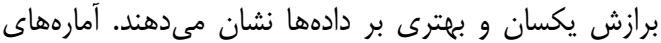
اكاييك،

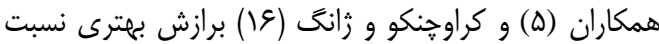

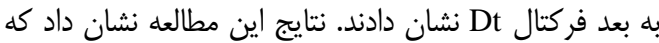

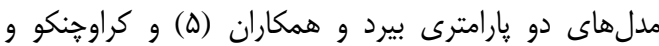

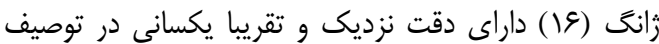

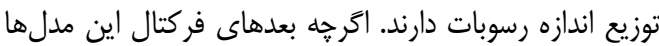

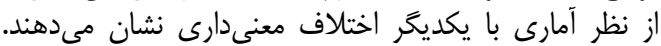

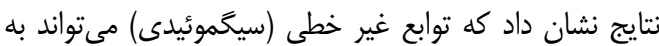

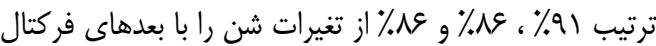

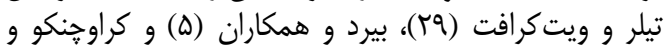

1. Ahmadi, A., M. Neyshabouri and H. Asadi. 2010. Relationship between fractal dimension of particle size distribution. Journal of water and soil, 20/1(4): 73-81 (In Persian).

2. Bayat, H., N. Davatgar and M. Jalali. 2014. Prediction of CEC using fractalparameters by artificial neural networks. Intenationa Agrophys, 28: 143-152(In Persian)

3. Bayat, H. 2008. Create transfer functions to predict retention curve through artificial neural networks using fractal parameters and principal component analysis. Ph.D. Thesis, University of Tabriz Tabriz Iran, $180 \mathrm{pp}$ (In Persian).

4. Bazoubandi, M. 2012. Evaluation of land use patterns and its optimization (Case of study: Goleroudbar Watershed). M.Sc. Thesis Faculty of Desert study University of Semnan. Semnan, Iran. 110 pp (In Persian).

5. Bird, N.R.A., E. Perrier and M. Rieu. 2000. The water retention function for a model of soil structure with pore and solid fractal distributions. European Journal of Soil Science, 51: 55-63.

6. Buchan, G.D. 1989. Applicability of the simple lognormal model to particle-size distribution in soils Soil Science, 147: 155-161.

7. Buchan, G.D., K.S.Grewal and A.B. Robson. 1993. Improvedmodelsof particle-size distribution: An illustration of model comparisontechniques. Soil Science Society ofAmerica Journal, 57: 901-908.

8. Burnham, K.P. and D.R. Anderson. 2002. Model selection and multi-model inference: a practical information-theoretic approach. Springer, $206 \mathrm{pp}$.

9. Campbell, G.S. 1985. Soil physics with BASIC: Transport models for soil-plant Systems vol (14). Elsevier, Amsterdam, 167 pp.

10. Ersahin, S., H. Gunal, T. Kutlu, B. Yetgin and S. Coban. 2006. Estimating specific surface area and cation exchange capacity in soils using fractal dimension of particle-size distribution Geoderma, 136: 588-597.

11. Filgueira, R.R., L.L. Fournier, I.C. Cecilia, P. Crlati and M.G. Carcia. 2006.Particle-size distribution in soils: A critical study of the fractal model validation. Geoderma, 134: 327-334.

12. Fredlund, M.D., D.G. Fredlund and G.W. Wilson. 2000. An equationto represent grainsize distribution. Canadian Geotechnical Journal, 37: 817-827.

13. Gee, G.W., J. W. Bauder and A. Klute. 1986. Particle-size analysis Methods of soil analysis Part 1. Physical and mineralogical methods, 383-411.

14. Hassanli, A.M., A. Esmaeli Nameghi and S. Beecham. 2009. Evaluation of the effect of porous check dam location on fine sediment retention (a case study). Environ Monit Assess, 152: 319-32. 
15. Hwang, S.I., KP. Lee, D.S. Lee and S.E. Powers. 2002. Models for estimating soil particle-size distributions. Soil Science Society of America Journal, 66: 1143-1150.

16. Kravchenko, A. and R. Zhang. 1998. Estimating the soil water retention from particle-size distributions: A fractal approach, Soil Science, 163: 171-179.

17. Mehdizadeh, L., F. Asadzadeh and A. Samadi. 2015. Application of mathematical models to describe the particle size distribution of sediments behind successive check dams. Journal of Watershed Engineering and Management, 4: 323-336 (In Persion).

18. Milla'n, H., M. Gonza'lez-Posada and M.J. Aguilar. 2003. On the fractal scaling of soil data Particlesize distributions. Geoderma, 117: 117-128.

19. Mohammadi, M.H., M. Khatar and M. Vanclooster. 2014. Combining a single hydraulic conductivity measurement with particle size distribution data for estimating the full range partially saturated hydraulic conductivity curve. Soil Science Society of America Journal, 78: 1594-1605.

20. Nadeu, E., A.A. Berhe, J.de Vente and C. Boix-Fayos. 2012. Erosion, deposition and replacement of soil organic carbon in Mediterranean catchments: a geomorphological, isotopic and land use change approach. Bio Geosciences, 9: 1099-1111.

21. Lee, T.K. and H.M. Ro. 2014. Estimating soil water retention function from its particle-size distribution Geosciences Journal, 18: 219-230.

22. Rousseva, S.S. 1997. Data transformations between soil textureschemes European Journal of Soil Science, 48: 749-758

23. SAS Institute. 2004. User's guide version 9.1: Statistics SAS Institute, Cary, NC.

24. Shiozawa, S. and G.S. Campbell. 1991. On the calculation of mean particle diameter and standard deviation from sand, silt, and clay fractions. Soil science, 152: 427-431.

25. Su, Y.Z., H.L. Zhao, W.Z. Zhao and T.H. Zhang. 2004. Fractal features of soilparticle size distribution and the implication for indicating desertification. Geoderma, 122: 43-49.

26. Tirgarsoltani, M.T., A.A. Zolfaghari, M. Gorji and M. Shorafa. 2012. Investigatepractical constraints functions ofdescribing soil particle size distribution.Journal of Soil andWater, 26: 67-76 (In Persian).

27. The Math Works, Inc. 2007. MATLAB: the language of technical computing. version 7.5.

28. Tirgarsoltani, M.T., M. Gorji, M.H. Mohammadi and H. Millan. 2014. Evaluation of models for description of wet aggregate size distribution from soils of different land uses. Soil Science Plant Nutrition, 60: 123-133.

29. Tyler, SW. and S.W. Wheatcraft. 1992. Fractal scaling of soil particle-size distributions: analysis and limitations. Soil Science Society of America Journal, 56: 362-369.

30. Tyler, SW. and S.W. Wheatcraft. 1989. Application of fractal mathematics to soil water retention estimation .Soil Science Society of America Journal, 53: 987-996.

31. Xiao, L., X. Sha, L. GuoBin and Z. Chao. 2014. Fractal features of soil profiles under different land use patterns on the Loess Plateau, China. Journal of Arid Land, 6: 550-560.

32. Zar, J.H. 1999. Biostatistical analysis Pearson Education, London, UK 663 pp.

33. Zhao, P., M. Shao and J. Zhuang. 2009. Fractal features of particle size redistributions of deposited soils on the dam farmlands. Soil Science, 174: 403-407.

34. Zhao, P. M. Shao and R. Horton. 2011. Performance of soil particle-size distribution Models for Describing Deposited Soils Adjacent to Constructed Dams in the China Loess Plateau Acta Geophysica, 59: 124-138.

35. Zolfaghari, A.A., M.T. Tirgarsoltani, M.R. Yazdani and E. Solimamni. 2014. Evaluation of efficiency models to describing soil particle size distribution Journal of Water and Soil, 45: 199-209 (In Persian). 


\title{
Evaluation of Fractal Models in Describing Particle Size Distribution of Sediment (Case of study: Fooladmahale of Semnan)
}

\section{Haniye Babrnejad Ziarat ${ }^{1}$, Ali Asghar Zolfaghari ${ }^{2}$, Mohammd Reza Yazdani ${ }^{3}$, Ali Asghar Hashemi' and Mohamad Kia Kiyaniyan}

\author{
1, 3 and 5- M.Sc. Student, Associate Professor and Instructor, of Faculty of Desert science, Semnan University \\ 2- Assistance Professor, of Faculty of Desert science, Semnan University, Semnan, Iran \\ 4- Assistance professor, Natural Resources Research Center of Semnan \\ (Corresponding Author: azolfaghari@semnan.ac.ir) \\ Received: March 9, $2015 \quad$ Accepted: April 11, 2016
}

\begin{abstract}
The objective of current study was to invstigate the accuracy of Tyler and Wheatcraft, Bird et al. and Kravchenko and Zhang fractal models in describing particle size distribution (PSD) of sediment in 14 successive check dams for two depths of 0-15 and 15-30 cm. Sediment PSD were determined in 28 samples using hydrometer method. The referred fractal models were fitted on sediments PSD data. Results indicated that there was no regular relationamong fractal dimensions insuccessive check dams. In addition, sediments transport from surroundingsoils was more observable in check dams. Two-parameter models of Bird et al. and Kravchenko and Zhang presented a better fit compared to one-parameter model of Tyler and Wheatcraft. The results of statistical analysis proved that the sigmoid function was able to describe the relation between fractal dimensions and clay, sand and silt percentage with a greater accuracy compared to the linear sigmoid function. Stepwise regressionanalysisdetermined astrong and significant correlation $\left(\mathrm{R}^{2}=0.97 * *\right)$ between fractal dimensionobtained from Tyler and Wheatcraft modelwithclay and sandcontents. According the result of this study, the determination of sediment PSD is possible bymeasurement of clayandsand amount.
\end{abstract}

Keywords: Check Dams, Fractal Dimensions, Sigmoid Function 